\title{
Evaluation of the Trends of Stomach Cancer Incidence in Districts of Iran from 2000-2010: Application of a Random Effects Markov Model
}

\author{
Farid Zayeri $^{1}$,Anita mansouri ${ }^{1}$,Ali Sheidaei ${ }^{1}$,Shadi Rahimzadeh ${ }^{2}$, Nazila Rezaei ${ }^{2}$, \\ Mitra Modirian², Sara khademioureh ${ }^{2}$, Ahmad Reza Baghestani' ${ }^{1}$, Farshad \\ Farzadfar $^{2 *}$
}

\begin{abstract}
Background: Stomach cancer is the fifth most common cancer and the third leading cause of death among cancers throughout the world. Therefore, stomach cancer outcomes can affect health systems at the national and international levels. Although stomach cancer mortality and incidence rates have decreased in developed countries, these indicators have a raising trend in East Asian developing countries, particularity in Iran. In this study, we aimed to determine the time trend of age-standardized rates of stomach cancer in different districts of Iran from 2000 to 2010. Materials and Methods: Cases of cancer were registered using a pathology-based system during 2000-2007 and with a population-based system since 2008 in Iran. In this study, we collected information about the incidence of stomach cancer during a 10 year period for 31 provinces and 376 districts, with a total of 49,917 cases. We employed two statistical approaches (a random effects and a random effects Markov model) for modeling the incidence of stomach cancer in different districts of Iran during the studied period. Results: The random effects model showed that the incidence rate of stomach cancer among males and females had an increasing trend and it increased by 2.38 and 0.87 persons every year, respectively. However, after adjusting for previous responses, the random effects Markov model showed an increasing rate of 1.53 and 0.75 for males and females, respectively. Conclusions: This study revealed that there are significant differences between different areas of Iran in terms of age-standardized incidence rates of stomach cancer. Our study suggests that a random effects Markov model can adjust for effects of previous responses.
\end{abstract}

Keywords: Stomach neoplasms - incidence - trends - Iran - random effects Markov model

Asian Pac J Cancer Prev, 17 (2), 661-665

\section{Introduction}

Cancer, which is the second most common cause of death in many developed countries and the third one in some developing countries, is an important global public health concern (Bener et al., 2008; Herszenyi and Tulassay, 2010). According to GLOBOCAN estimates, cancer led to about 14.1 million of new cases and about 8.2 million deaths in 2012. Stomach cancer, with roughly 950,000 new cases and 723,000 deaths in 2012, is the fifth most common cancer and the third leading cause of death among cancers throughout the world (Cancer, 2014; Hu et al., 2015). Therefore, stomach cancer outcomes can affect the health systems and result in a significant burden; it can affect different dimensions of health at the national and international levels (Shrivastava et al., 2015).

Although stomach cancer mortality and incidence rates have decreased in developed countries (Colquhoun et al., 2015; Torre et al., 2015), these indicators have a rising trend in East Asian developing countries (Eichelberger et al., 2015). In view of that, about two-thirds of stomach cancer cases occur in Asian people (Colquhoun et al., 2015). More specifically, stomach cancer with about 7300 cases per year is the third most common cancer in Iran which is a developing country located in southwest Asia (Movahedi et al., 2009). In other words, Iran has the highest rate of stomach cancer among Middle East countries (Mohagheghi et al., 2009). Studies conducted on Iranian population showed that the Age-Standardized Incidence Rates (ASIR) of stomach cancer has increased from 11.37 per 100000 in 2003 to 15.21 per 100000 in 2006 for males and from 5.20 per 100000 to 6.89 per 100000 for females in the mentioned period (Mousavi et al., 2009). 
The wide variation in geographical distribution of stomach cancer incidence and mortality rates is the main characteristic of this cancer in Iran (Malekzadeh et al., 2009). For instance, ASIR in northwestern provinces of Iran (Ardebil: 49.1 for males and 25.4 for females) are higher than that in the southern regions (Kerman: 10.2 for males and 5.1 for females) (Malekzadeh et al., 2009; Movahedi et al., 2009; Almasi et al., 2015). However, it is suggested that variations in incidence and mortality rates are due to differences in dietary patterns, environmental risk factors, availability of fresh products, and lifestyle (Torre et al., 2015). In view of that, studying trends of stomach cancer incidence and mortality in different regions could provide an opportunity for health policy makers to monitor risk factors of this cancer and make purposive decisions about distribution of health services in each geographical area.

Many studies have been previously conducted about stomach cancer epidemiology and its risk factors in different regions of Iran (Sadjadi et al., 2003; Behrouzian and Aghdami, 2004; Alireza et al., 2005; Yazdizadeh et al., 2005; Samadi et al., 2007; Malekzadeh et al., 2009; Mohagheghi et al., 2009; Mousavi et al., 2009; Movahedi et al., 2009; Radmard, 2010; Almasi et al., 2015; Amin et al., 2015; Baeradeh et al., 2015; Khaleghian et al., 2015). However, our literature review showed that no comprehensive time trend study has been conducted yet to assess the stomach cancer incidence rates in different districts of Iran. With regards to the lack of related studies in one side and the importance of evaluation of the time trend of the cancer incidence on the other side, we decided to explore age-standardized time trend pattern of the cancer incidence among both sexes in different districts of Iran from 2000 to 2010 . To do this, we applied random effects and random effects Markov models for modeling our longitudinal data.

\section{Materials and Methods}

\section{Data source and data preparation}

The cases of cancer are mostly registered via a pathology-based system in Iran. According to the regulations designed by the Ministry of Health and Medical Education (MOHME), all pathology labs are obliged to regularly report new cases of cancer to medical universities. The MOHME collects the related data from all universities at the end of each year. Using IARC software, data sets are combined by cancer registry office in MOHME. After primary processes, this office makes the data available for researchers. In our study, we used stomach cancer incidence data set collected from 2000 to 2010 . We did not access the data about the year 2006 because this data set was not validated. Therefore, in this study the data about incidence of stomach cancer collected during 10 years. This data set included 31 provinces, 376 districts, and 49917 cases.

First, all individuals with more than 15 years of age were selected. Annual crude incidence rates were calculated in all districts by sex. A direct method of age standardization was used to adjust the effect of variations in age distribution across districts.

\section{Statistical analysis}

As mentioned there is an almost high variation of stomach cancer incidence rates in Iran. Thus, to achieve the applicable and realistic results it is necessary to control several sources of variation. On the other hand, the simple and easy to interpret models are advised. Therefore, we suggested a random effect approach to model the trend of stomach cancer incidence in Iran.

Random effect models are able to capture unobserved individual heterogeneity (Diggle et al., 2002). Since in our study we defined districts as individuals, many sources of heterogeneity in districts were covered in random effect component of the model. One of the most important sources of heterogeneity could be the percentage of incomplete data at each district. Incompleteness not only differed from a district to another one but also was changing over time. It was the main idea for introducing the model below with random effect of year:

$$
Y_{i t}=\beta_{0}+\beta_{1} \text { year }+b_{i} \text { year }+e_{i t}
$$

Where $Y_{i t}$ and $e_{i t}$ indicate ASIR and error term for district $i$ in year $t$. Also, $b \sim N\left(0, \sigma_{b}^{2}\right)$ and $e_{i t} \sim N\left(0, \sigma_{b}^{2}\right)$ are random component and error term which have normal distributions (Diggle et al., 2002).

Although random effects model is able to control heterogeneity sources, it does not consider all types of contagion. The contagion is a critical characteristic of longitudinal analysis that is ignored in most of applications. Aitkin and Alfo 2003 studied this concept. It is consisted of true and apparent contagion. Apparent contagion occurs when individuals are drawn from heterogeneous population and are captured by the random effect model (Aitkin and Alfo, 2003).

As mentioned above, there is another type of contagion called true contagion. It is based on the assumption which says future outcomes are directly influenced by the past values (Aitkin and Alfo, 2003). As an example, in our study, the incidence rate in a specific year depends on the incidence rate of the preceding years. The best approach to deal with this phenomenon is to consider lag responses in the model. Therefore, we suggest an alternative model based on random effect component and lag responses that called random effect transition model:

$$
Y_{i t}=\beta_{0}+\beta_{1} \text { year }+\beta_{2} Y_{i(t-1)}+b_{i} \text { year }+e_{i t}
$$

Where $\mathrm{Y}_{\mathrm{i}(\mathrm{t}-1)}$ denotes the value of response in one time before the present response.

We fitted both suggested models to compare the results obtained from the two approaches. In both models $\beta_{1}$ is the slope of changes in ASIR of stomach cancer calculated for every new year. When true contagion is negligible, it is expected to have equal $\beta_{1}$ estimated by the two models. On the other hand, in the presence of true contagion, the results of random effects model could be misleading.

\section{Results}

Evaluation of ASIR of stomach cancer showed a 
Stomach Cancer Incidence Trends in Districts of Iran from 2000-2010: Application of a Random E Markov Model

rising trend in majority of districts along the years of the study. Table 1 presents a summary of information about the minimum, maximum, median, mean and standard deviation of incidence rates per 100,000 persons for all districts of Iran from 2000 to 2010. According to this table, national ASIR increased from 24.8 in 2000 to 42.9 in 2010 for males and from 20.8 to 22.8 for females in the same period. In addition, mean and median of incidence rates of stomach cancer, respectively, were 16.47 and 12.23 for males in 2000 and increased to 36.71 and 32.72 in 2010. In addition, the mean incidence rate was 9.30 in 2000 and
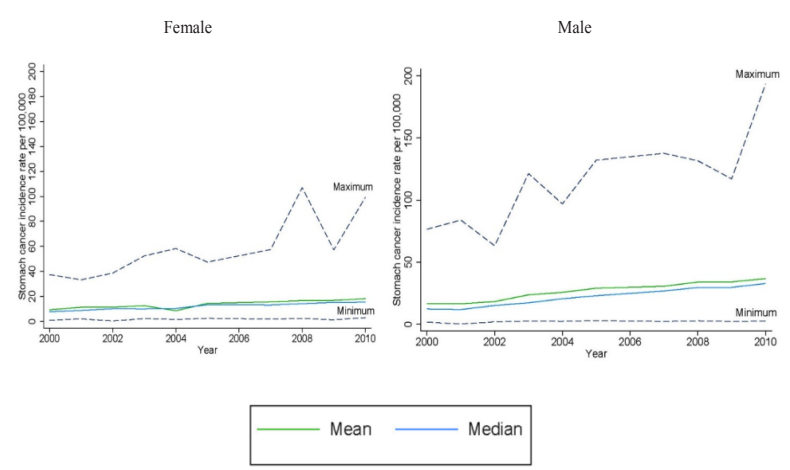

Figure 1. Stomach Cancer Incidence Rate Per 100,000 During 2000-2010
18.17 in 2010 for females and its median was 7.70 and 15.34 , respectively.

Differences in incidence rates between males and females strongly support the idea of higher incidence rate of stomach cancer among males than females. For instance, mean incidence rate for males in 2010 was 2.02 times more than that for females in the same year. However, this ratio was not constant for all the studied years. As another example, the incidence rate was 2.99 in 2004. For more clarification mean, median, maximum and minimum of age standardized incidence rates for both sexes are illustrated in Figure 1.

As shown in Table 1, there is a relatively high variation in stomach cancer incidence in Iran districts. It is supported by high values of standard deviation of incidence rates. The standard deviation of incidence rates was $65.57 \%$ in males and $69.24 \%$ in females in 2010 . Finally, of the medians were lower than the means in all years except for females in 2004 (the median was 1.81 case per 100,000 persons higher than the mean). Therefore, the distribution of ASIR of stomach cancer is right skewed.

We aimed to detect changes in cancer stomach incidence rates based on the specifications of each district. Estimated coefficients of year in fitted models that were explained in method section could be interpreted as the

Table 1. Descriptive Analysis about Age Standardized Incidence Rate of Stomach Cancer in Iran Per 100,000 Population

\begin{tabular}{ccccll}
\hline Gender & ASIR* & Mean \pm SD & Median & \multicolumn{1}{c}{ Minimum (District -Province) } & Maximum (District-Province) \\
\hline Male & & & & & \\
2000 & 24.8 & $16.47 \pm 13.78$ & 12.23 & 1.62 (Karaj-Alborz) & 76.44 (Qazvin-Qazvin) \\
2001 & 29.6 & $16.38 \pm 14.85$ & 11.76 & 0.13 (Tehran-Tehran) & 83.85 (Qazvin-Qazvin) \\
2002 & 30.4 & $18.19 \pm 12.87$ & 15.05 & 1.84 (Marand -East-Azar) & 63.17 (Meshkinshahr-Ardebil) \\
2003 & 42.1 & $23.61 \pm 19.99$ & 17.17 & 2.43 (Minab-Hormozgan) & 121.31 (Maku-West-Azar) \\
2004 & 42.9 & $25.61 \pm 19.25$ & 20.49 & 2.32 (Maragheh-East-Azar) & 96.88 (Gachsaran-Kohgiluyeh) \\
2005 & 44.5 & $28.94 \pm 20.68$ & 22.89 & 2.84 (Nikshahr-Sistan) & 132.16 (Meshkinshahr-Ardebil) \\
2007 & 45.8 & $30.53 \pm 20.21$ & 26.72 & 2.21 (Baharestan-Tehran) & 137.61 (Maku-West-Azar) \\
2008 & 45.9 & $33.91 \pm 22.62$ & 29.51 & 2.68 (Dashtestan-Boshehr) & 131.68 (Robatkarim-Tehran) \\
2009 & 42.9 & $34.03 \pm 21.00$ & 29.68 & 2.22 (Bandare-e-Mahshahr-Khozestan) & 117.04 (Robatkarim-Tehran) \\
2010 & 42.9 & $36.71 \pm 24.07$ & 32.72 & 2.41 (Lar-Fars) & 193.4 (Robatkarim-Tehran) \\
Female & & & & & \\
2000 & 20.8 & $9.30 \pm 6.49$ & 7.7 & 0.97 (Tehran-Tehran) & 37.49 (Khoramabad-Lorestan) \\
2001 & 24.9 & $11.37 \pm 7.51$ & 8.79 & 1.99 (Shahr-e-Kord-Chaharmahal bakhtiari) & 33.26 (Salmas-West-Azar) \\
2002 & 17.8 & $11.41 \pm 7.32$ & 10.14 & 0.39 (Tabriz-East-Azar) & 38.67 (Tonekabon-Mazandaran) \\
2003 & 22.3 & $12.55 \pm 8.99$ & 10.06 & 2.19 (Varamin-Tehran) & 52.39 (Maku-West-Azar) \\
2004 & 22.5 & $8.56 \pm 1.57$ & 10.37 & 1.57 (Eslamshahr-Tehran) & 58.32 (Maku-West-Azar) \\
2005 & 23.2 & $14.43 \pm 8.21$ & 13.34 & 2.43 (Maragheh-East-Azar) & 47.48 (Darreshahr-Ilam) \\
2007 & 23.1 & $15.66 \pm 9.10$ & 13.14 & 1.81 (Bojnurd-North-Khorasan) & 57.52 (Meshkinshahr-Ardebil) \\
2008 & 25.3 & $16.68 \pm 12.03$ & 14.23 & 2.35 (Dashtestan-Boshehr) & 107.03 (Robatkarim-Tehran) \\
2009 & 22.1 & $16.85 \pm 10.32$ & 15.25 & 1.32 (Malayer-Hamadan) & 57.24 (Maku-West-Azar) \\
2010 & 22.8 & $18.17 \pm 12.58$ & 15.34 & 3.01 (Bandar-e-Mahshahr-Khozestan) & 99.33 (Kalat-Khorasan-e-Razavi) \\
\hline
\end{tabular}

*Age Standardized incidence Rate of stomach cancer in Iran per 100,000 population

Table 2. Random Effects and Random Effects Markov Models Results

\begin{tabular}{|c|c|c|c|c|c|}
\hline Gender & Model & Variable & Estimate $\pm \mathrm{SD}$ & P-value & AIC \\
\hline \multirow[t]{3}{*}{ Male } & Random effects model & Year & $2.38 \pm 0.10$ & $<0.001$ & 22311.6 \\
\hline & Random effects Markov model & Year & $1.53 \pm 0.13$ & $<0.001$ & 19089.2 \\
\hline & & Lag response & $0.34 \pm 0.02$ & $<0.001$ & \\
\hline \multirow[t]{3}{*}{ Female } & Random effects model & Year & $0.87 \pm 0.06$ & $<0.001$ & 15019.1 \\
\hline & Random effects Markov model & Year & $0.75 \pm 0.08$ & $<0.001$ & 12564.3 \\
\hline & & Lag response & $0.19 \pm 0.03$ & $<0.001$ & \\
\hline
\end{tabular}


main aim of this study. Therefore, we summarized the results of both models for both sexes in Table 2. As shown, Akaike's information criterion (AIC) for the models with lag responses is considerably lower than simply random effect model. In males, AIC of random effects model was 22311.6 while AIC of random effects Markov model was 19089.2. The difference between AIC values in females was 2454.8 .

In addition to the better fit, random effects Markov model lead to a more realistic coefficient for the slope of trend. The random effects model showed that the incidence rate of stomach cancer for males and females, respectively, increased by 2.38 and 0.87 person every year. However, after adjusting for the previous responses, the random effects Markov model showed an increasing rate of 1.53 and 0.75 for men and women, respectively.

\section{Discussion}

In this study, we showed that the incidence of stomach cancer rose from 2000 to 2010 in different districts of Iran. Using simple random effect model, the slopes of ASIR changes were estimated as 2.38 and 0.87 in males and females, respectively. Using the random effect Markov model, these values were estimated as 1.53 and 0.75 , respectively. Considering the significant differences between estimated values and goodness of fit criteria, we concluded that true contagion affected the results. Therefore, we suggest results of random effects Markov model which can adjust the effect of this type of contagion and achieve more inferences that are reliable.

The review of literature on stomach cancer in Iran showed that incidence of this neoplasm has had an increasing trend during recent years. Almasi et al. in 2005(Almasi et al., 2015), Enayatrad and Salehiniya in 2014 (Enayatrad and Salehiniya, 2014), and Najafi et al. in 2011 (Najafi et al., 2011) reported the increasing trends in national and some subnational areas. In addition, in line with the global findings (Brenner et al., 2009; Cancer, 2014; Colquhoun et al., 2015; Torre et al., 2015), Etemad and Gooya in 2009 (Agahjani H, 2008) in a study on Iranian population reported that the incidence of stomach cancer in males is twice more than that in females. Our results are in agreement with these studies. In addition, we also found that slope of changes in incidence rates in males was two times more than that in females.

Considering the geographical distribution of stomach cancer incidence in Iran, it seems that the northern and northwestern areas had higher incidence rates, the central and western areas had medium incidence rates, and the southern regions had low incidence rates. Malekzadeh et al. in 2009 (Malekzadeh et al., 2009) conducted a review study and investigated the incidence rates of stomach cancer in different areas of Iran. Based on their study, Ardabil in northwest of Iran had the highest ASIR with 49.1 and 25.4 per 100,000 population for males and females, respectively (Sadjadi et al., 2003). In Tehran, as a province that is located in the central part of Iran, the ASIR was calculated as 19.8 and 10.0 for males and females, respectively (Mohagheghi et al., 2009). Our results are in line with the results of previous studies about the geographical pattern of stomach cancer ASIR in districts level. In contrast, some districts in provinces with high ASIR had low ASIR; on the other hand, some districts with high ASIR were located in low risk provinces. These contradictions provide a strong reason to explore stomach cancer ASIR at district level instead of province or national level. In addition, the descriptive statistics presented in Table 1 verified our hypothesis to conduct analysis at districts level. For instance, the range of ASIRs was relatively wide and had a large standard deviation in comparison with the mean ASIRs.

As mentioned, the cancer registry in Iran is conducted mostly via a pathology-based system. This kind of registration is less efficient than population-based registration. This limitation may affect results of every study conducted on cancer. Lack of effective covariates in our models was another likely limitation of our study. In addition, there are several more complex statistical models which could cover and investigate spatial correlation and time effect simultaneously. We did not use these models because of computational limitations. As we tried to control the effect of incompleteness of cancer registration system via introducing a random effect component, future research is recommended to calculate and apply the incompleteness values.

Eventually, this study revealed that there are remarkable differences in ASIR of stomach cancer among different areas of Iran. These differences can be observed not only in provinces but also in districts. The mentioned differences not only leads to an increase in the trend of stomach cancer ASIR, but also highlights the need for making a comprehensive and purposeful plan to control stomach cancer in all districts.

\section{References}

Agahjani H EtK, Gooya MM (2008). National report on registered cancer cases in 2008. Tehran, Iran: Cancer Office, Centre for Disease Control, Deputy for Health, Ministry of Health and Medical Education.

Aitkin M, Alfò M (2003). Longitudinal analysis of repeated binary data using autoregressive and random effect modelling. Statistical Modelling, 3, 291-303.

Alireza S, Mehdi N, Ali M, et al (2005). Cancer occurrence in Iran in 2002, an international perspective. Asian Pac J Cancer Prev, 6, 359.

Almasi Z, Rafiemanesh H, Salehiniya H (2015). Epidemiology characteristics and trends of incidence and morphology of stomach cancer in iran. Asian Pac J Cancer Prev, 16, 2757.

Amin M, Kazemi A, Eskandari O, et al (2015). Geographical distribution of stomach cancer related to heavy metals in Kurdistan, Iran. Int J Environ Health Engineering, 4, 12.

Baeradeh NA, Lotfi MH, Fallahzadeh H, et al (2015). Survival rate of patients with stomach cancer and its effective factors in yazd province.

Behrouzian R, Aghdami N (2004). Urinary iodine/creatinine ratio in patients with stomach cancer in Urmia, Islamic Republic of Iran. East Mediterr Health J, 10, 921-4.

Bener A, Ayub H, Kakil R, et al (2008). Patterns of cancer incidence among the population of Qatar: a worldwide comparative study. Asian Pac J Cancer Prev, 9, 19-24.

Brenner H, Rothenbacher D, Arndt V (2009). Epidemiology of stomach cancer. in 'Cancer Epidemiology', Eds Springer, 

467-77

Cancer IAfRo (2014). World cancer report 2014. Geneva: WHO.

Colquhoun A, Arnold M, Ferlay J, et al (2015). Global patterns of cardia and non-cardia gastric cancer incidence in 2012. Gut, gutjnl-2014-308915.

Diggle P, Heagerty P, Liang KY, et al 2002. Analysis of longitudinal data, Oxford University Press.

Eichelberger L, Murphy G, Etemadi A, et al (2015). Risk of Gastric Cancer by Water Source: Evidence from the Golestan Case-Control Study. PloS one, 10, 128491.

Enayatrad M, Salehiniya H (2014). Trends in gastric cancer incidence in Iran. J Mazandaran Univ Med Sci, 24, 8-16.

Herszenyi L, Tulassay Z (2010). Epidemiology of gastrointestinal and liver tumors. Eur Rev Med Pharmacol Sci, 14, 249-58.

$\mathrm{Hu}$ J, La Vecchia C, Negri E, et al (2015). Macronutrient intake and stomach cancer. Cancer Causes Control, 26, 839-47.

Khaleghian M, Jahanzad I, Shakoori A, et al (2015). C-MYC amplification and expression in stomach cancer samples in Iranian population using two techniques of CISH and IHC. Tehran University Med J, 73, 260-70.

Malekzadeh R, Derakhshan MH, Malekzadeh Z (2009). Gastric cancer in Iran: epidemiology and risk factors. Arch Iran Med, 12, 576-83.

Mohagheghi MA, Mosavi-Jarrahi A, Malekzadeh R, et al (2009). Cancer incidence in tehran metropolis: the first report from the tehran population-based cancer registry. Arch Iranian $\mathrm{Med}, \mathbf{1 2}, 15-23$.

Mousavi SM, Gouya MM, Ramazani R, et al (2009). Cancer incidence and mortality in Iran. Ann Oncol, 20, 556-63.

Movahedi M, Afsharfard A, Moradi A, et al (2009). Survival rate of gastric cancer in Iran. $J$ Res Med Sci, 14, 367.

Najafi F, Mozaffari H, Karami M, et al (2011). Trends in incidence of gastrointestinal tract cancers in Western iran, 1993-2007. Iranian Red Crescent Med J, 13, 805.

Radmard AR (2010). Five common cancers in Iran. Archives Iranian Med, 13, 143-6.

Sadjadi A, Malekzadeh R, Derakhshan MH, et al (2003). Cancer occurrence in ardabil: results of a population-based cancer registry from Iran. Int J Cancer, 107, 113-8.

Samadi F, Babaei M, Yazdanbod A, et al (2007). Survival rate of gastric and esophageal cancers in Ardabil province, NorthWest of Iran. Arch Iran Med, 10, 32-7.

Shrivastava S, Shrivastava P, Ramasamy J (2015). Formulating a comprehensive public health strategy for the prevention and control of stomach cancer. J Med Tropics, 17, 46.

Torre LA, Bray F, Siegel RL, et al (2015). Global cancer statistics, 2012. CA Cancer J Clin, 65, 87-108.

Yazdizadeh B, Jarrahi AM, Mortazavi H, et al (2005). Time trends in the occurrence of major GI cancers in Iran. Asian Pac J Cancer Prev, 6, 130-4. 